\section{A maxillary sinus mass}

A 55-year-old Chinese woman presented to the ear, nose and throat clinic with a 6-month history of left-sided blood-stained mucus. It was dark brown with no epistaxis. There was no mucopus, nasal obstruction, facial pain or fever. She denies any history of hypertension, trauma, head and neck cancer, anticoagulation use or previous irradiation. Physical examination did not reveal any external nasofacial swelling or erythema, and extraocular movement was intact. Nasoendoscopy revealed a fleshy mass with overlying blood vessels arising above the left inferior turbinate, with septal deviation towards the right (Fig. 1).

A well-circumscribed lesion was seen in the left maxillary sinus on T2-weighted (Fig. 2) and T1-weighted magnetic resonance imaging (MRI) with contrast (Fig. 3), extending into the left nasal cavity with mass effect. The contents had serpiginous areas of marked enhancement, suggestive of areas of heterogeneous soft tissue density with interspersed blood vessels. As seen in Fig. 2, a T2-weighted MRI also depicts a hypointense peripheral rim. On computed tomography (CT) paranasal sinuses (Fig. 3), complete opacification of the left maxillary sinus is seen with medial and superior expansion with severely attenuated bony walls, particularly along the displaced left medial orbital floor and medial sinus wall, as well as a right septal deviation with no invasion into adjacent structures.

What is the diagnosis?
A. Maxillary sinus haematoma
B. Capillary haemangioma
C. Maxillary sinus carcinoma
D. Mucocoele
E. Angiofibroma

A fleshy red mass with a thin fibrous covering was seen intraoperatively. Frozen section performed revealed mainly red blood cells and acute inflammatory exudates.

Discussion. Organised haematoma of maxillary sinus (OHMS), commonly known as maxillary sinus haematoma, is a benign haemorrhagic pseudotumour. The number of reported cases is fewer than 100 cases. $^{1}$

Ozaki et al. postulated the "Negative Spiral Theory" that illustrates how an OHMS is formed. Firstly, haemorrhage occurs in the maxillary sinus due to various causes, such as an underlying haemangioma, coagulopathy, trauma, radiation therapy, infection or

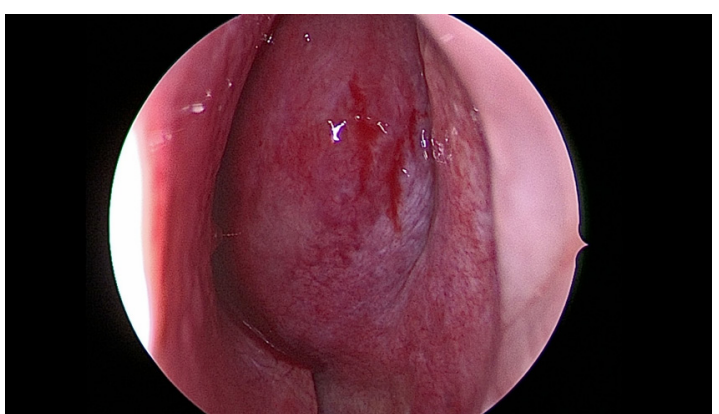

Fig. 1. Nasoendoscopic image of a nasal tumour with surface vessels.

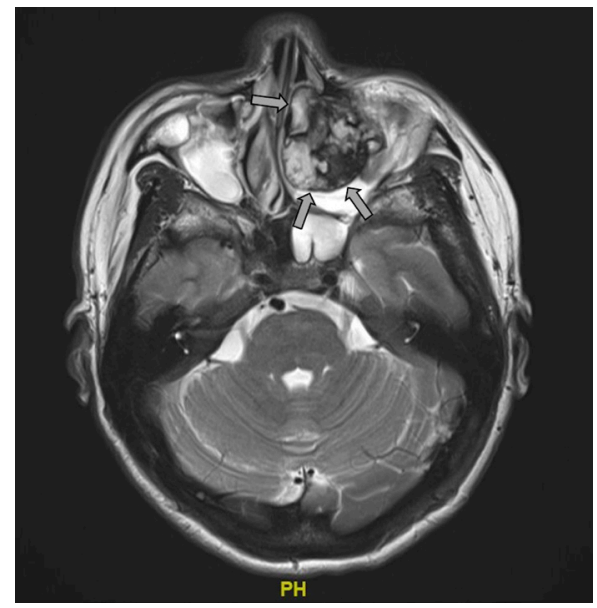

Fig. 2. Axial view of T2-weighted magnetic resonance imaging. The grey arrows illustrate a hypointense peripheral rim suggestive of a fibrous pseudocapsule.

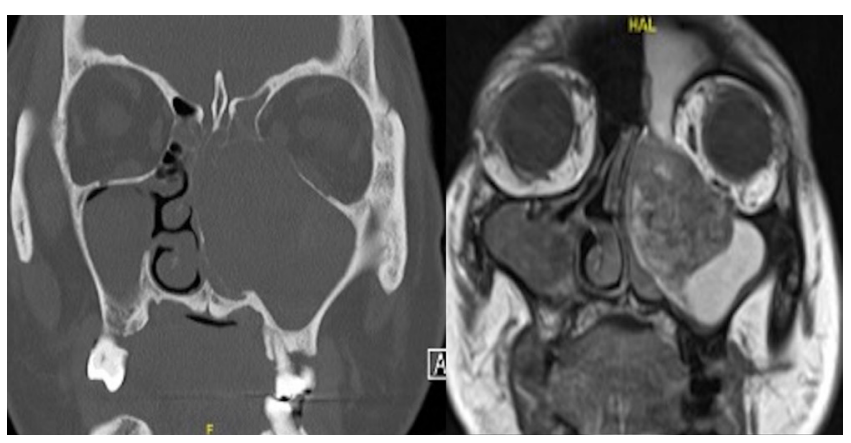

Fig. 3. Coronal views of computed tomography paranasal sinuses bone window and T1-weighted magnetic resonance imaging with contrast.

inflammation. When necrosis and inflammation occurs, a fibrous capsule forms in the enclosed space, preventing the resorption of inflammatory cells and blood. Subsequently, 
hyalinisation and angiogenesis recur. As the cycle continues, the OHMS enlarges, causing mass effect and pressure erosion on surrounding structures. ${ }^{2}$

The diagnosis of OHMS described by Urata et al. consists of 4 criteria: (1) findings of nasal bleeding or obstruction and a unilateral, polypoidal nasal mass; (2) CT revealing an expanding maxillary lesion with thinning or destruction of surrounding bony wall; (3) MRI showing a heterogeneous mass; and (4) histopathological findings of dilated vessels, haemorrhage and fibrin exudation. ${ }^{3}$

OHMS are asymptomatic when localised to the maxillary sinus. Symptoms occur when they expand, erode and displace adjacent structures. Risk factors include an underlying haemangioma, bleeding diathesis, previous trauma, radiation, infection or inflammation, hypertension or anticoagulation use. Symptoms include epistaxis, unilateral nasal congestion, cheek swelling, retroorbital pain and blurring of vision. Nasoendoscopy may show a fleshy tumour with overlying telangiectasia. Gross examination may reveal ipsilateral proptosis and hypoesthesia over the ipsilateral maxillary region. ${ }^{4}$

It is important to note that OHMS is not typically a top differential diagnosis in a middle-aged patient presenting with a unilateral sinonasal mass with no history of trauma or anticoagulation use.

Radiologically, OHMS has a mixture of marked heterogeneous hypo-intensity and iso-intensity on MRI, surrounded by a hypo-intense peripheral rim, which matches histologically with a fibrous pseudocapsule. These findings reflect the histological heterogeneity of the lesion, which comprises haemorrhage, fibrosis and neovascularisation. ${ }^{5}$

Haemangiomas can be divided into capillary or cavernous types. The former may show marked early enhancement with subsequent washout. They are described as well-circumscribed masses with no internal calcification and homogeneous enhancement. ${ }^{6}$ Cavernous haemangiomas are usually large, inhomogeneous masses with a heterogeneous enhancement pattern of either a centripetal or multifocal nodular pattern.?

Sinonasal cavernous haemangiomas and OHMS can appear similar radiologically, and some believe that these entities are different manifestations of the same disease. Others are of the view that OHMS and cavernous haemangiomas are distinct entities because the vascular lumina of cavernous haemangiomas are histologically larger than those of OHMS. It is postulated that an OHMS associated with a sinonasal polyp may be a special form of angiomatous polyp, though the pathogenesis is not clearly understood. ${ }^{5}$
While imaging features may overlap between haemangioma, angiofibroma and OHMS, the age and sex of the patient are atypical for a haemangioma and angiofibroma. Haemangiomas are the most common head and neck tumours in children, but rarely occur in the sinonasal cavity. ${ }^{6}$ The mass is also more anteriorly centred in the left maxillary sinus whereas an angiofibroma typically occurs around the sphenopalatine foramen, and almost always occur in young men. Enhancement in a large cavernous haemangioma or angiofibroma may be more marked than what is seen in this lesion. Mucocoeles are typically non-enhancing. Carcinoma has a solid pattern of nodular enhancement and typically shows invasive features extending into surrounding soft tissue as well as the ipsilateral ethmoid sinuses. ${ }^{8}$

Histopathological confirmation is required to rule out differentials. Histopathology for this patient revealed blood and fibrinous material, haemorrhagic and inflamed granulation tissue with underlying fibrosis, chronic inflammation and ectatic vessels with organising haematoma, consistent with the description of OHMS in literature.

Management of OHMS typically involves surgical resection, which is diagnostic and curative should the mass be resected completely. Bleeding diathesis must be optimised as OHMS may be haemorrhagic and cause massive bleeding. Both open and endoscopic approaches have been employed in the removal of this haematoma. Given its benign condition, most lesions are well circumscribed rather than infiltrative, and can be adequately addressed by an endoscopic middle meatal antrostomy. ${ }^{9,10}$

\section{REFERENCES}

1. Almasoud M, Alhumaidan A, Ashoor M. Maxillary sinus hematoma: Current pathogenesis and management. Egyptian Journal of Ear, Nose, Throat and Allied Sciences 2014;15:37-40.

2. Ozaki M, Sakai S, Ikeda H. Hemangioma of the nasal cavity and sinuses-a report of twenty five cases. Otolaryngol Head Neck Surg (Tokyo). 1977;49:8.

3. Urata S, Ohki M, Tsutsumi T, et al. Organised haematoma of the maxillary sinus: pathophysiological differences suggesting a new aetiological hypothesis. J Laryngol Otol 2013;127:519-24.

4. Tabaee A, Kacker A. Hematoma of the maxillary sinus presenting as a mass--a case report and review of literature. Int J Pediatr Otorhinolaryngol 2002;65:153-7.

5. Kim E, Kim H, Chung S, et al. Sinonasal Organized Hematoma: CT and MR Imaging Findings. Am J Neuroradiol 2008;29:1204-8.

6. Dillon W, Som P, Rosenau W. Hemangioma of the nasal vault: MR and CT features. Radiology 1991;180:761-5. 
7. Kim H, Kim J, Kim J, et al. Bone erosion caused by sinonasal cavernous hemangioma: CT findings in two patients. Am J Neuroradiol 1995;16:1176-8.

8. Lee H, Smoker W, Lee B. Organized Hematoma of the Maxillary Sinus: CT Findings. Am J Roentgenol 2007;188:370-3.

9. Yokoi H, Arakawa A, Matsumoto F, et al. Organized hematoma of the maxillary sinus: a clinicopathologic study of 5 cases. Ear Nose Throat J 2014;93:23-6.

10. Imayoshi S, Kanazawa $\mathrm{T}$, Fukushima $\mathrm{N}$ et al. Three Cases of Organized Hematoma of the Maxillary Sinus: Clinical Features and Immunohistological Studies for Vascular Endothelial Growth Factor and Vascular Endothelial Growth Factor Receptor 2 Expressions. Case Rep Otolaryngol 2015;2015:846832.
Kelvin Yong Jie $\underline{\operatorname{Lim}}^{1}$, Siu Cheng $\underline{\text { Loke }}^{2}$ FRCR, Jian Li Tan ${ }^{1}$ FAMS ORL, Ming Yann $\underline{\operatorname{Lim}}^{1}$ FAMS ORL

${ }^{1}$ Department of Otorhinolaryngology, Tan Tock Seng Hospital, Singapore ${ }^{2}$ Department of Diagnostic Radiology, Tan Tock Seng Hospital, Singapore

Correspondence: Dr Kelvin Yong Jie Lim, Department of Otorhinolaryngology, Tan Tock Seng Hospital, 11 Jln Tan Tock Seng, Singapore 308433 .

Email: kelvin.lim@mohh.com.sg 